ESAIM: COCV 19 (2013) 516-532

DOI: $10.1051 / \mathrm{cocv} / 2012019$
ESAIM: Control, Optimisation and Calculus of Variations

www.esaim-cocv.org

\title{
MINIMIZING THE FUEL CONSUMPTION OF A VEHICLE FROM THE SHELL ECO-MARATHON: A NUMERICAL STUDY*
}

\author{
SOPHIE JAN ${ }^{1}$
}

\begin{abstract}
We apply four different methods to study an intrinsically bang-bang optimal control problem. We study first a relaxed problem that we solve with a naive nonlinear programming approach. Since these preliminary results reveal singular arcs, we then use Pontryagin's Minimum Principle and apply multiple indirect shooting methods combined with homotopy approach to obtain an accurate solution of the relaxed problem. Finally, in order to recover a purely bang-bang solution for the original problem, we use once again a nonlinear programming approach.
\end{abstract}

Mathematics Subject Classification. 49J15, 90C90, 49N90.

Received June 1st, 2011. Revised January 16, 2012.

Published online February 21, 2013.

\section{INTRODUCTION}

In the context of environment preservation, Shell company organizes each year a competition named Shell Eco-marathon. The aim is to design and build a vehicle, and to optimize the driving strategy in order to use as little fuel as possible to travel a given distance $L$ in a time less than a given duration $T$.

For example, for a competition on the race track displayed in Figure $1, L \approx 25.5 \mathrm{~km}$ (7 laps) and $T \approx 51 \mathrm{~min}$. This quantity $T$ corresponds to the time needed to travel the distance $L$ with a mean speed of $30 \mathrm{~km} / \mathrm{h}$.

This paper is organized in four sections. In Section 1, we describe the problem and formulate it as an intrinsically bang-bang optimal control problem. Then, we analyze, in Section 2, a relaxed version of this optimal control problem using Pontryagin's Minimum Principle. In the third section, we report numerical experiments on both the relaxed and the original optimal control problems. Finally, we conclude in Section 4.

\section{Position of the PROBlem}

In this section, we first describe the vehicle and the race track. Then we make explicit the dynamics of the vehicle, and we introduce some scaling quantities that are numerically interesting. Finally, we model the problem of minimizing the fuel consumption of the vehicle as an optimal control problem.

\footnotetext{
Keywords and phrases. Optimal control, singular arcs, nonlinear programming, continuation method, indirect multiple shooting.

* This work has been partially supported by French National Research Agency (ANR) through COSINUS program (project ID4CS No. ANR-09-COSI-005).

1 Université de Toulouse \& CNRS, Institut de Mathématiques de Toulouse, UMR 521931062 Toulouse Cedex 9, France.

sophie.jan@math.univ-toulouse.fr
} 

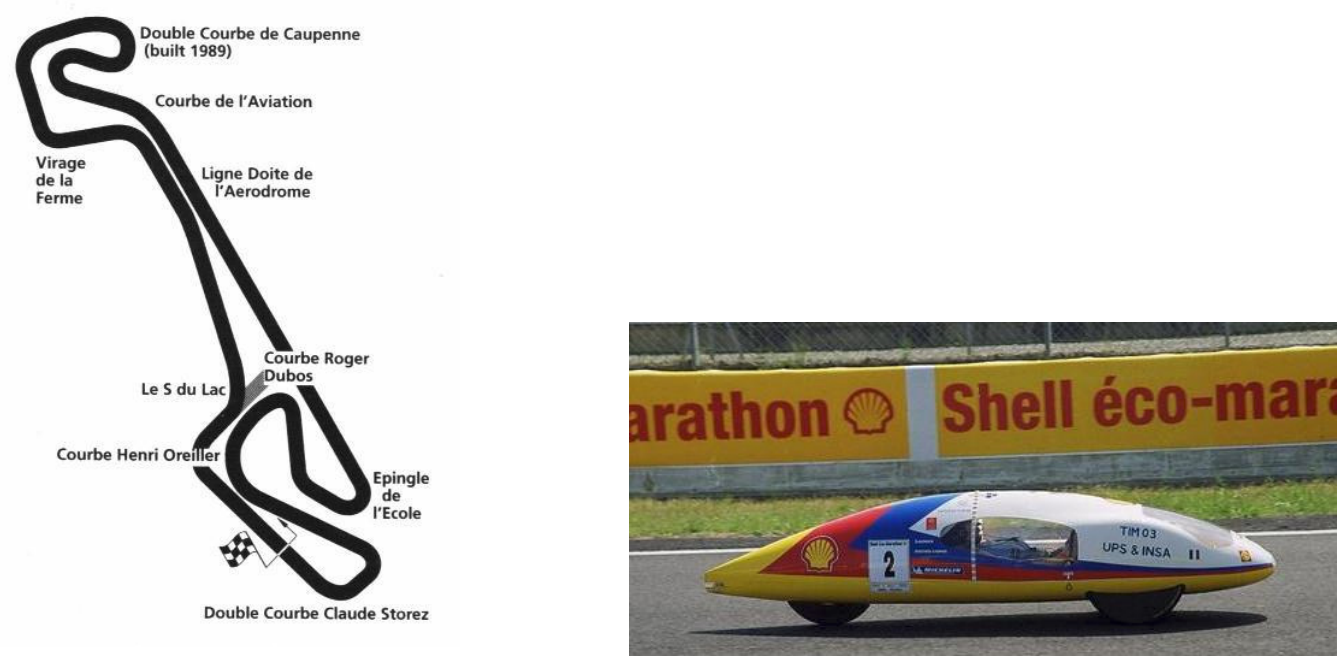

FiguRE 1. Left: Nogaro's race circuit (South-West of France). Right: the vehicle of the Toulouse Ingénierie Mécanique team (Université Paul Sabatier and Institut National des Sciences Appliquées de Toulouse).

\subsection{The vehicle}

The vehicle is represented in Figure 1. It has three identical wheels, two at the front and one at the rear, whose radius and moment of inertia are respectively denoted by $R$ and $I_{r}$. We assume that the engine is well-balanced and $\Gamma$ denotes its torque. We denote by $C_{x}$ and $S$ the drag coefficient and frontal surface of the vehicle. The quantity $M$ depicts the total mass of the vehicle and its pilot.

In the sequel, we use $d(t)$ and $v(t)$ to denote respectively the distance covered by the vehicle from the starting point and its speed at time $t$. We use $k$ to represent the friction coefficient of the wheels on the road. It seems physically quite reasonable to consider $k$ as a function of the speed of the vehicle $v$. However, in this study, we shall assume that the friction coefficient $k(v)$ is essentially constant, equal to a certain $k_{0}$. Since $k(v)$ is physically zero when the speed is zero, the function $k(v)$ is assumed to be a function of class $\mathcal{C}^{2}$ that satisfies the following constraints:

$$
k(0)=0, \quad k(v)=k_{0} \text { for all } v \geq \varepsilon, \quad k^{\prime}(\varepsilon)=0, \quad k^{\prime \prime}(\varepsilon)=0
$$

for some given $\varepsilon$. The choice of $\varepsilon$ is discussed in Section 3.2. The representative curve of $k$ is displayed in Figure 2 .

In the eco-marathon competition, the allowed driving strategy consists in alternating periods where the engine is "on" with periods where it is off. We use the variable $u(t)$ to describe this strategy: $u(t)$ is equal to one when the engine is "on", and it is zero otherwise.

\subsection{The race track}

The profile of the race track is only known through altitudes measured at some points. With these tabulated data, we build a function $\alpha$ of class $\mathcal{C}^{2}$ using piecewise-cubic spline interpolation. The value $\alpha(d)$ represents the angle between the horizontal axis and the slope of the road when the vehicle has covered a distance $d$ on the race track. 


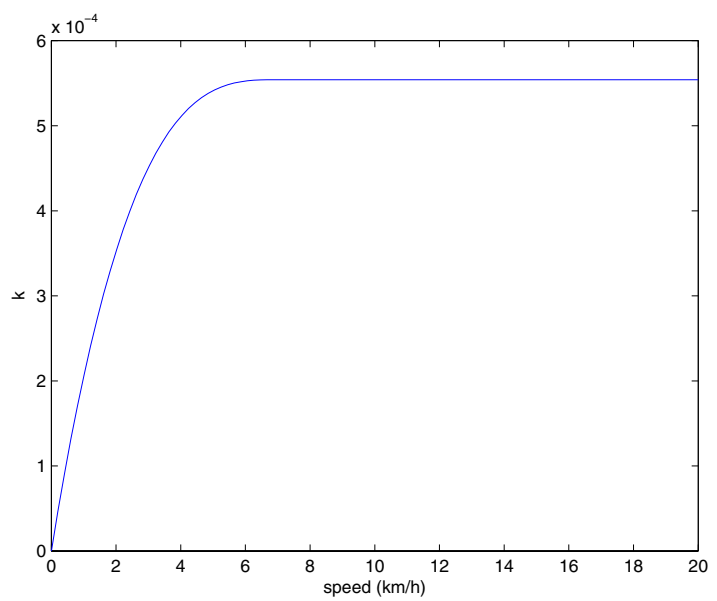

FiguRE 2. Representative curve of the function $k(v)$.

\subsection{Dynamics of the vehicle}

We describe now the dynamics of the vehicle using the second Newton's law in the longitudinal direction. This gives, for all $t>0$,

$$
\begin{array}{rlrl}
\left(\frac{3 I_{r}}{R^{2}}+M\right) \dot{v}(t)= & -M g \sin (\alpha(d(t))) & & \text { (gravitation) } \\
& -\frac{1}{2} \rho C_{x} S v(t)^{2} & & \text { (aerodynamics) } \\
& -M g k(v(t)) \cos (\alpha(d(t))) & \text { (rolling friction) } \\
& +u(t) \frac{\Gamma}{R} & & \text { (engine) }
\end{array}
$$

where $g$ is the gravity constant and $\rho$ is the air density.

Then, denoting

$$
A=\frac{3 I_{r}}{R^{2}}+M>0, B=-\frac{\rho C_{x} S}{2 A}<0, C=-\frac{M g}{A}<0, D=\frac{\Gamma}{R A}>0,
$$

and

$$
\mathcal{C}(d, v)=C(\sin (\alpha(d))+k(v) \cos (\alpha(d)))
$$

we may rewrite the preceding equation under the form of the following system of differential equations of order one:

$$
\left(\begin{array}{c}
\dot{d}(t) \\
\dot{v}(t)
\end{array}\right)=\left(\begin{array}{c}
v(t) \\
B v(t)^{2}+\mathcal{C}(d(t), v(t))+D u(t)
\end{array}\right) .
$$

\subsection{Rescaling}

For numerical purposes, we perform the following rescaling:

$$
t=t_{c} \tau, \quad d(t)=x_{c} \delta(\tau), \quad v(t)=v_{c} \nu(\tau), \quad u(t)=U(\tau),
$$

where $\tau \in[0,1]$ and where $t_{c}:=T, x_{c}$ and $v_{c}$ are characteristic quantities of time, length and speed (respectively). 
With these new variables, the equations for the dynamics become

$$
\left(\begin{array}{c}
\dot{\delta}(\tau) \\
\dot{\nu}(\tau)
\end{array}\right)=\left(\begin{array}{c}
\tilde{A} \nu(\tau) \\
\tilde{B} \nu(\tau)^{2}+\tilde{\mathcal{C}}(\delta(\tau), \nu(\tau))+\tilde{D} U(\tau)
\end{array}\right)
$$

where

$$
\tilde{A}:=\frac{t_{c} v_{c}}{x_{c}}, \quad \tilde{B}:=B v_{c} t_{c}, \quad \tilde{\mathcal{C}}(\delta, \nu):=\frac{t_{c}}{v_{c}} \mathcal{C}\left(x_{c} \delta, v_{c} \nu\right), \quad \tilde{D}:=D \frac{t_{c}}{v_{c}} .
$$

Let us also introduce the following notations:

$$
t_{f}=t_{c} \tau_{f}, \quad T=t_{c} \Upsilon, \quad L=x_{c} \Delta .
$$

\subsection{The optimal control problem}

The eco-marathon challenge can be modeled under the form of the following optimal control problem (Lagrange form):

$$
\begin{aligned}
& \text { Minimize fuel consumption }:=\int_{0}^{\tau_{f}} U(\tau) \nu(\tau) \mathrm{d} \tau \\
& \text { subject to } 0 \leq \tau_{f} \leq \Upsilon \text {, } \\
& U(\tau) \in\{0,1\} \quad \forall \tau \in\left[0, \tau_{f}\right] \\
& \delta(0)=0, \quad \nu(0)=0, \\
& \forall \tau \in\left[0, \tau_{f}\right],\left\{\begin{array}{l}
\dot{\delta}(\tau)=\tilde{A} \nu(\tau), \\
\dot{\nu}(\tau)=\tilde{B} \nu(\tau)^{2}+\tilde{\mathcal{C}}(\delta(\tau), \nu(\tau))+\tilde{D} U(\tau),
\end{array}\right. \\
& \delta\left(\tau_{f}\right)=\Delta \text {, }
\end{aligned}
$$

where the unknowns are the function $U$ and the final time $\tau_{f}$.

This problem may be reformulated into the following Mayer form:

$$
\begin{aligned}
& \text { Minimize fuel consumption }:=X_{3}\left(\tau_{f}\right) \\
& \text { subject to } 0 \leq \tau_{f} \leq \Upsilon, \\
& \\
& U(\tau) \in\{0,1\} \quad \forall \tau \in\left[0, \tau_{f}\right] \\
& X(0)=(0,0,0), \\
& \left.\forall \tau \in] 0, \tau_{f}\right], \dot{X}(\tau)=f(X(\tau), U(\tau)), \\
& \\
& X_{1}\left(\tau_{f}\right)=\Delta,
\end{aligned}
$$

where

$$
\begin{aligned}
X:[0,1] & \rightarrow \mathbb{R}^{3} \\
\tau & \mapsto(\delta(\tau), \nu(\tau), e(\tau)),
\end{aligned}
$$

and

$$
f(X, U)=\left(\begin{array}{c}
\tilde{A} \nu \\
\tilde{B} \nu^{2}+\tilde{\mathcal{C}}(\delta, \nu)+\tilde{D} U \\
U \nu
\end{array}\right) .
$$

In our application context, for $\tau \in\left[0, \tau_{f}\right] \subset[0,1]$, it is obvious that both the distance $\delta$ covered by the vehicle and its speed $\nu$ are bounded. Then, it is immediate to prove, using the Cauchy-Lipschitz theorem [10], that there exists a unique solution for the problem:

$$
\begin{aligned}
& X(0)=(0,0,0), \\
& \left.\forall \tau \in] 0, \tau_{f}\right], \dot{X}(\tau)=f(X(\tau), U(\tau)) .
\end{aligned}
$$


We study the following relaxed optimal control problem:

$$
\begin{aligned}
& \text { Minimize fuel consumption }:=X_{3}\left(\tau_{f}\right) \\
& \text { subject to } 0 \leq \tau_{f} \leq \Upsilon \text {, } \\
& U(\tau) \in[0,1] \quad \forall \tau \in\left[0, \tau_{f}\right], \\
& X(0)=(0,0,0), \\
& \left.\forall \tau \in] 0, \tau_{f}\right], \dot{X}(\tau)=f(X(\tau), U(\tau)), \\
& X_{1}\left(\tau_{f}\right)=\Delta \text {, }
\end{aligned}
$$

where the control function $U$ now takes values in the interval $[0,1]$. This "continuous relaxation" means that we are not restricted to on/off engine switches anymore: we are also allowed to accelerate more or less.

It seems natural that the final time $t_{f}$ (respectively $\tau_{f}$ ) be equal to the maximal authorized time $T$ (respectively $\Upsilon$ ). This will be numerically demonstrated in Section 4.1 .

As a consequence, in the next section, we shall analyse the relaxed optimal control problem (2.3) assuming that the final time $\tau_{f}$ is fixed and equal to $\Upsilon$.

\section{AnAlysis of the optimal CONTROL PROBLEM}

In this section, we analyze the following problem using Pontryagin's Minimum Principle:

$$
\begin{aligned}
& \text { Minimize the consumption }:=X_{3}(\Upsilon) \\
& \text { subject to } U(\tau) \in[0,1] \quad \forall \tau \in[0, \Upsilon], \\
& \\
& X(0)=(0,0,0), \\
& \forall \tau \in] 0, \Upsilon], \dot{X}(\tau)=f(X(\tau), U(\tau)), \\
& \\
& X_{1}(\Upsilon)=\Delta .
\end{aligned}
$$

Assuming that we are not in a pathological case, the Hamiltonian associated with Problem (3.1) is defined by

$$
H(X, U, \Lambda):=p \tilde{A} \nu+q\left(\tilde{B} \nu^{2}+\tilde{\mathcal{C}}(\delta, \nu)+\tilde{D} U\right)+r U \nu,
$$

where $\Lambda=(p, q, r)$ represents the vector of adjoint variables or costates.

Consequently, the adjoint equations are

$$
\dot{\Lambda}(\tau)=g(X(\tau), U(\tau), \Lambda(\tau)),
$$

where

$$
g(X, U, \Lambda):=-\left(\begin{array}{c}
q \frac{\partial \tilde{\mathcal{C}}}{\partial \delta}(\delta, \nu) \\
p \tilde{A}+q\left(2 \tilde{B} \nu+\frac{\partial \tilde{\mathcal{C}}}{\partial \nu}(\delta, \nu)\right)+r U \\
0
\end{array}\right)
$$

\subsection{Application of Pontryagin's minimum principle}

Applying Pontryagin's Minimum Principle [3-6,11], we have that a necessary condition for $(X(),. U()$.$) being$ a solution of (3.1) is the existence of a piecewise smooth vector $\Lambda():.=(p(),. q(),. r()$.$) such that:$

1. $(X(),. \Lambda()$.$) solves (2.2)$ and $(3.2)$;

2. $\forall U \in[0,1], H(X(\tau), U(\tau), \Lambda(\tau)) \leq H(X(\tau), U, \Lambda(\tau))$, for almost every $\tau \in[0, \Upsilon]$;

3. $\Lambda() \neq$.0 ;

4. $q(\Upsilon)=0$, and $r(\Upsilon)=1$. 
Using the so-called transversality condition $r(\Upsilon)=1$ and the third equation of (3.2), we have that

$$
r(\tau)=1, \forall \tau \in[0, \Upsilon]
$$

Since the Hamiltonian is linear with respect to the control, we have

$$
H(X, U, \Lambda):=\beta(X, \Lambda) U+\gamma(X, \Lambda),
$$

with $\beta(X, \Lambda):=\nu+\tilde{D} q$ and $\gamma(X, \Lambda):=\tilde{A} p \nu+q\left(\tilde{B} \nu^{2}+\tilde{\mathcal{C}}(\delta, \nu)\right)$. We obtain easily that an optimal control for (3.1) is given by

$$
U(\tau)=\left\{\begin{array}{l}
0 \text { if } \beta(X(\tau), \Lambda(\tau))>0 \\
1 \text { if } \beta(X(\tau), \Lambda(\tau))<0
\end{array}\right.
$$

The function $\Psi(\tau):=\beta(X(\tau), \Lambda(\tau))$ is called the switching function.

\subsection{Singular arcs}

We analyze now the case where the switching function vanishes on a closed interval $\left[\tau_{0}, \tau_{1}\right] \subset[0, \Upsilon]$ :

$$
\Psi(\tau):=\nu(\tau)+\tilde{D} q(\tau)=0, \forall \tau \in\left[\tau_{0}, \tau_{1}\right] .
$$

In order to compute the singular control, we use a classical technique, recalled in [3] for example: we differentiate relation (3.3) with respect to time until $U$ appears explicitly.

Remark 3.1. For the sake of notational simplicity, we do not write explicitly the dependence on $\tau$ in the sequel of this section.

First, we have $\dot{\Psi}(\tau)=0$ for almost every $\tau \in\left[\tau_{0}, \tau_{1}\right]$. This gives, using the Hamiltonian equations (2.2)-(3.2) and relation (3.3),

$$
\begin{aligned}
0 & =\dot{\Psi} \\
& =\dot{\nu}+\tilde{D} \dot{q} \\
& =\tilde{B} \nu^{2}+\tilde{\mathcal{C}}(\delta, \nu)+\tilde{D} U-\tilde{D}\left(U+p \tilde{A}+q\left(2 \tilde{B} \nu+\frac{\partial \tilde{\mathcal{C}}}{\partial \nu}(\delta, \nu)\right)\right) \\
& =\tilde{B} \nu^{2}+\tilde{\mathcal{C}}(\delta, \nu)-\tilde{D} \tilde{A} p+\nu\left(2 \tilde{B} \nu+\frac{\partial \tilde{\mathcal{C}}}{\partial \nu}(\delta, \nu)\right) \\
& =3 \tilde{B} \nu^{2}+\tilde{\mathcal{C}}(\delta, \nu)-\tilde{D} \tilde{A} p+\nu \frac{\partial \tilde{\mathcal{C}}}{\partial \nu}(\delta, \nu) .
\end{aligned}
$$

This relation does not depend on the control $U$.

We differentiate once more to obtain, almost everywhere on $\left[\tau_{0}, \tau_{1}\right]$ :

$$
\begin{aligned}
0 & =\ddot{\Psi} \\
& =6 \tilde{B} \nu \dot{\nu}+\frac{\partial \tilde{\mathcal{C}}}{\partial \delta}(\delta, \nu) \dot{\delta}+\frac{\partial \tilde{\mathcal{C}}}{\partial \nu}(\delta, \nu) \dot{\nu}-\tilde{A} \tilde{D} \dot{p}+\dot{\nu} \frac{\partial \tilde{\mathcal{C}}}{\partial \nu}(\delta, \nu)+\nu\left(\frac{\partial^{2} \tilde{\mathcal{C}}}{\partial \nu \partial \delta}(\delta, \nu) \dot{\delta}+\frac{\partial^{2} \tilde{\mathcal{C}}}{\partial \nu^{2}}(\delta, \nu) \dot{\nu}\right) \\
& =\left(\frac{\partial \tilde{\mathcal{C}}}{\partial \delta}(\delta, \nu)+\nu \frac{\partial^{2} \tilde{\mathcal{C}}}{\partial \nu \partial \delta}(\delta, \nu)\right) \dot{\delta}-\tilde{A} \tilde{D} \dot{p}+\left(6 \tilde{B} \nu+2 \frac{\partial \tilde{\mathcal{C}}}{\partial \nu}(\delta, \nu)+\nu \frac{\partial^{2} \tilde{\mathcal{C}}}{\partial \nu^{2}}(\delta, \nu)\right) \dot{\nu}
\end{aligned}
$$

Using now the Hamiltonian equations and relation (3.3), we have

$$
\begin{aligned}
0 & =\ddot{\Psi} \\
& =\left(\frac{\partial \tilde{\mathcal{C}}}{\partial \delta}(\delta, \nu)+\nu \frac{\partial^{2} \tilde{\mathcal{C}}}{\partial \nu \partial \delta}(\delta, \nu)\right) \tilde{A} \nu+\tilde{A} \tilde{D} q \frac{\partial \tilde{\mathcal{C}}}{\partial \delta}(\delta, \nu)+\left(6 \tilde{B} \nu+2 \frac{\partial \tilde{\mathcal{C}}}{\partial \nu}(\delta, \nu)+\nu \frac{\partial^{2} \tilde{\mathcal{C}}}{\partial \nu^{2}}(\delta, \nu)\right)\left(\tilde{B} \nu^{2}+\tilde{\mathcal{C}}(\delta, \nu)+\tilde{D} U\right) \\
& =\left(\left(6 \tilde{B}+\frac{\partial^{2} \tilde{\mathcal{C}}}{\partial \nu^{2}}(\delta, \nu)\right) \nu+2 \frac{\partial \tilde{\mathcal{C}}}{\partial \nu}(\delta, \nu)\right)\left(\tilde{B} \nu^{2}+\tilde{\mathcal{C}}(\delta, \nu)+\tilde{D} U\right)+\tilde{A} \nu^{2} \frac{\partial^{2} \tilde{\mathcal{C}}}{\partial \nu \partial \delta}(\delta, \nu)
\end{aligned}
$$


The way the parameter $\varepsilon$ has been chosen in the definition of the function $k$ renders negative the first factor in (3.5) for all $\delta \in[0, \Delta]$, for all $\nu \in\left[0, \frac{3 v_{m}}{v_{c}}\right]\left(v_{m}\right.$ is the mean speed of $\left.30 \mathrm{~km} / \mathrm{h}\right)$. Thus, the Legendre-Clebsch necessary optimality condition for the existence of a singular arc is satisfied [8]. More precisely:

$$
-\frac{\partial}{\partial U}\left(\frac{\mathrm{d}^{2}}{\mathrm{~d} t^{2}} \frac{\partial H}{\partial U}\right) \geq 0
$$

Using (3.4) and (3.3), we first obtain, almost everywhere on $\left[\tau_{0}, \tau_{1}\right]$ :

$$
p(\tau)=\frac{3 \tilde{B} \nu(\tau)^{2}+\tilde{\mathcal{C}}(\delta, \nu)+\nu \frac{\partial \tilde{\mathcal{C}}}{\partial \nu}(\delta, \nu)}{\tilde{A} \tilde{D}} \text { and } q(\tau)=-\frac{\nu(\tau)}{\tilde{D}}
$$

We then derive $U$ by using (3.5):

$$
U(\tau)=-\frac{1}{\tilde{D}}\left(\frac{\tilde{A} \nu^{2} \frac{\partial^{2} \tilde{\mathcal{C}}}{\partial \nu \partial \delta}(\delta, \nu)}{\left(6 \tilde{B}+\frac{\partial^{2} \tilde{\mathcal{C}}}{\partial \nu^{2}}(\delta, \nu)\right) \nu+2 \frac{\partial \tilde{\mathcal{C}}}{\partial \nu}(\delta, \nu)}+\tilde{B} \nu(\tau)^{2}+\tilde{\mathcal{C}}(\delta, \nu)\right)
$$

\subsection{Summary}

A necessary condition for $(X(),. U()$.$) to be a solution of (3.1)$ is the existence of a nontrivial vector $\Lambda():.=$ $(p(),. q()$.$) such that Y():.=(X(),. \Lambda()$.$) is solution of$

$$
\dot{Y}(\tau)=h(Y(\tau), U(\tau))
$$

with

$$
h(Y, U)=\left(\begin{array}{c}
\tilde{A} \nu \\
\tilde{B} \nu^{2}+\tilde{C}(\delta, \nu)+\tilde{D} U \\
U \nu \\
-q \frac{\partial \tilde{\mathcal{C}}}{\partial \delta}(\delta, \nu) \\
-\left(\tilde{A} p(\tau)+q(\tau)\left(2 \tilde{B} \nu+\frac{\partial \tilde{\mathcal{C}}}{\partial \nu}(\delta, \nu)\right)+U\right)
\end{array}\right),
$$

with the two-point boundary conditions:

$$
Y_{1}(0)=0, Y_{2}(0)=0, Y_{3}(0)=0, Y_{1}(\Upsilon)=\Delta, Y_{5}(\Upsilon)=0,
$$

and

$$
U(\tau)= \begin{cases}0 & \left.\begin{array}{l}
\text { if } \Psi(\tau)>0, \\
-\frac{\tilde{A}}{\tilde{D}}\left(\frac{\partial^{2} \tilde{\mathcal{C}}}{\partial \nu \partial \delta}(\delta, \nu)\right. \\
1
\end{array}\right) \tilde{B} \nu(\tau)^{2}+\tilde{\mathcal{C}}(\delta, \nu) \\
\text { if } \Psi(\tau)=0 \text { for } \tau \in\left[\tau_{0}, \tau_{1}\right] \subset[0, \Upsilon], \\
\text { if } \Psi(\tau)<0,\end{cases}
$$

with the switching function $\Psi(\tau):=\nu(\tau)+\tilde{D} q(\tau)$. 
TABLE 1. Parameters of the numerical tests.

\begin{tabular}{|c|c|c|}
\hline \multicolumn{3}{|l|}{ Vehicle } \\
\hline Vehicle drag coefficient & & $C_{x}=0.1$ \\
\hline Frontal vehicle surface & $\mathrm{m}^{2}$ & $S=0.35$ \\
\hline Wheel inertia moment & $\mathrm{kg} \mathrm{m}^{2}$ & $I_{r}=0.041$ \\
\hline Wheel radius & $\mathrm{m}$ & $R=0.2375$ \\
\hline Vehicle (including pilot) mass & & $M=69$ \\
\hline Engine torque & $\mathrm{kg} \mathrm{m}^{2} \mathrm{~s}^{-2}$ & $\Gamma=5.5$ \\
\hline \multicolumn{3}{|l|}{ Race track } \\
\hline Mean speed & $\mathrm{m} \mathrm{s}^{-1}$ & $v_{m}=30 / 3.6\left(30 \mathrm{~km} \mathrm{~h}^{-1}\right)$ \\
\hline Distance to be covered & $\mathrm{m}$ & $L=3636$ (one lap) \\
\hline Maximum time allowed & $\mathrm{s}$ & $T=L / v_{m}$ \\
\hline \multicolumn{3}{|l|}{ Universal constants } \\
\hline Air density & $\mathrm{kg} \mathrm{m}^{-3}$ & $\rho=1.23$ \\
\hline Gravity constant & $\mathrm{m} \mathrm{s}^{-2}$ & $g=9.81$ \\
\hline
\end{tabular}

\section{Numerical EXPERIMENTS}

In this section, we apply the same techniques as in [3]. In a first step, in order to have an idea of the difficulty of the problem, we use a naive and simple nonlinear-programming approach. Then, since it is well known that indirect shooting methods are more accurate, we use such techniques to obtain more precise results. However, the major drawback of indirect methods lies in its initialization. That is why we combine indirect methods with a continuation process. Finally, we compare the preceding results with a purely bang-bang solution that we obtain via another nonlinear-programming approach.

To compute the results depicted in this section, we have considered the race track from Figure 1 and we have chosen the following characteristic quantities:

$$
t_{c}:=T, \quad x_{c}:=L \quad \text { and } \quad v_{c}:=v_{m},
$$

where $v_{m}$ is the mean speed of $30 \mathrm{~km} / \mathrm{h}$. With this choice, we have

$$
\Upsilon=1, \quad \text { and } \quad \Delta=1 .
$$

The other parameters defining our test problem here are summarized in Table 1 .

In the sequel, we report results for two cases: the general one and the simplified one. In the latter, we assume that the race track is flat. This means that the function $\alpha$ that represents the angle between the horizontal plane and the slope of the road is identically zero. Consequently, $\tilde{\mathcal{C}}$ only depends on $\nu$ :

$$
\tilde{\mathcal{C}}(\delta, \nu):=\frac{t_{c}}{v_{c}} C k\left(v_{c} \nu\right) .
$$

\subsection{A naive approach to solve the relaxed optimal control problem with $\tau_{f}$ free in $[0, \Upsilon]$}

In this section, we report results for the problem (2.3). First, we consider that the final time $\tau_{f}$ is free on the interval $[0, \Upsilon]$. Then, we discretize the time interval $\left[0, \tau_{f}\right]$ into $N$ subintervals of equal size $\Delta \tau:=\tau_{f} / N$, and we consider that the control remains constant on each of these subintervals. Let us set the following notation:

- $\tau_{i}:=i \Delta \tau$, for $i=0, \ldots, N$;

- $I_{i}:=\left[\tau_{i-1}, \tau_{i}\right]$, for $i=1, \ldots, N$; 

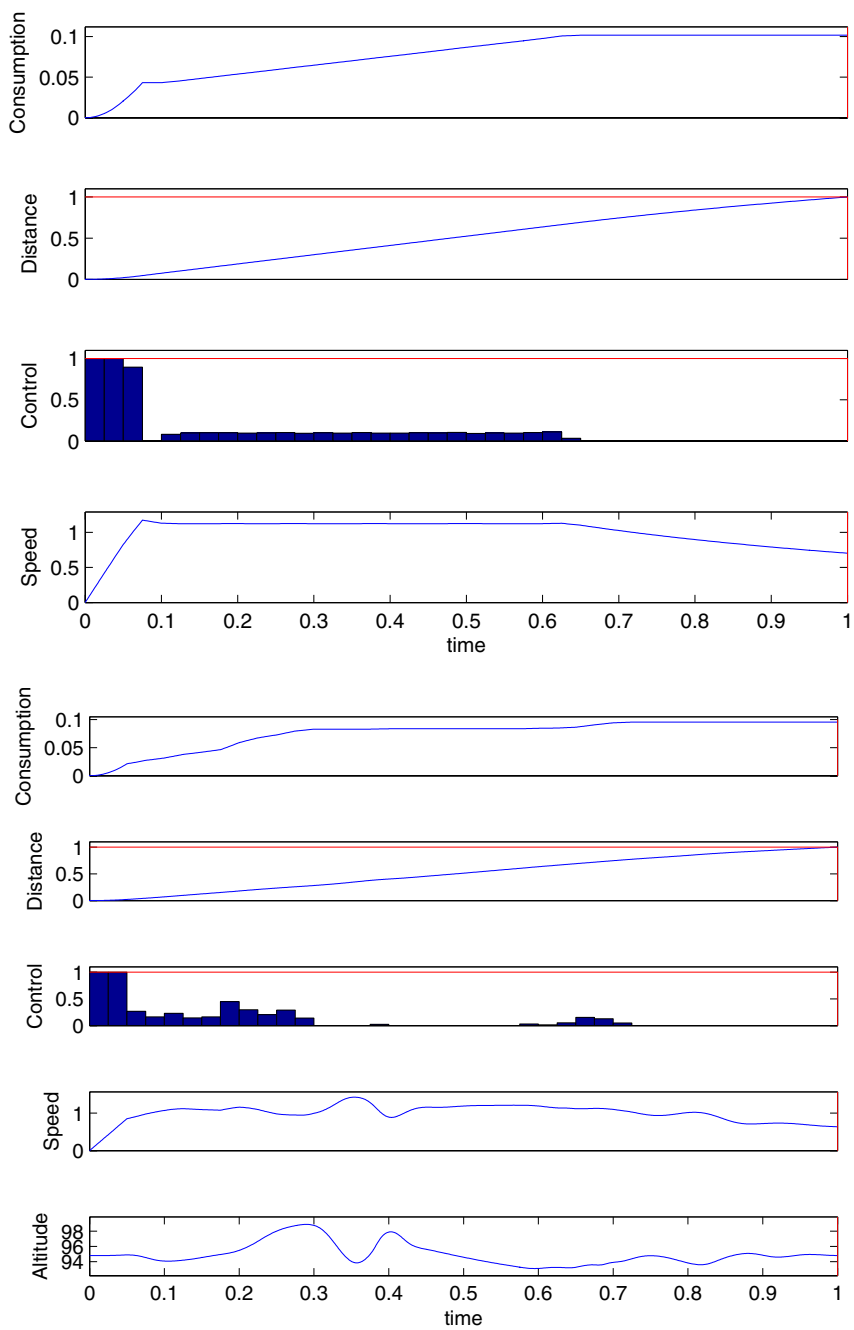

FiguRE 3. Results obtained by the naive nonlinear-programming approach (4.1) with $N:=$ 40 subintervals of $\left[0, \tau_{f}\right]$. Top: flat race track. Bottom: general case.

- $U_{i}$ is the value of the control on the subinterval $I_{i}$, for $i=1, \ldots, N$;

- $X(\tau, 1)$ is the solution of $(2.2)$ on $I_{1}$ with $U(\tau) \equiv U_{1}$ and $X\left(\tau_{0}, 1\right)=(0,0,0)$;

- $X(\tau, i)$ is the solution of $(2.2)$ on $I_{i}$ with $U(\tau) \equiv U_{i}$ and $X\left(\tau_{i-1}, i\right)=X\left(\tau_{i-1}, i-1\right)$ for $i=2, \ldots, N$.

Then, we consider the following problem:

$$
\begin{aligned}
\text { Minimize } & X_{3}\left(\tau_{N}, N\right) \\
\text { subject to } 0 \leq U_{i} \leq 1, i=1, \ldots, N, & \\
& 0 \leq \tau_{f} \leq \Upsilon \\
& X_{1}\left(\tau_{N}, N\right)=\Delta,
\end{aligned}
$$

where the unknowns are the $N$ values of $U_{i}$, and the value of the final time $\tau_{f}$.

To solve problem (4.1), we use the SQP fmincon (with the integrator ode113) routine of Matlab for various values of $N$, and various random initial guesses of the unknowns. Our results are displayed in Figure 3.

A first observation is that these results confirm numerically the fact that $\tau_{f}=\Upsilon$. Consequently, from now on, we assume that the final time $\tau_{f}$ is equal to $\Upsilon$ and we consider problem (3.1). 
These results also reveal the existence of singular arc(s). For the simplified case, we observe one singular arc. In the general case, it is more difficult to determine both the number of singular arcs, and their junction points with regular arcs.

\subsection{Structure of the control for the relaxed optimal control problem with $\tau_{f}=\Upsilon$}

Since we have numerically established in the preceding section that the final time is equal to the maximum authorized time, we concentrate now on problem (3.1). Our aim is to identify accurately the structure of the control solution for this problem. For this purpose, we use multiple indirect shooting methods. In order to avoid the well-known difficulties to initialize such indirect methods, we combine them with a continuation technique.

\subsubsection{Quadratic penalty}

Let us add to problem (3.1) a term quadratic in $U[3]$. We obtain, for $\lambda \in[0,1]$ :

$$
\begin{aligned}
& \text { minimize the consumption }:=X_{3}(\Upsilon) \\
& \text { subject to } U(\tau) \in[0,1] \quad \forall \tau \in[0, \Upsilon] \\
& \qquad(0)=(0,0,0), \\
& \forall \tau \in] 0, \Upsilon], \dot{X}(\tau)=f_{\lambda}(X(\tau), U(\tau)), \\
& X_{1}(\Upsilon)=\Delta,
\end{aligned}
$$

where

$$
f_{\lambda}(X, U)=f(X, U)+\left(\begin{array}{c}
0 \\
0 \\
(1-\lambda) U^{2}
\end{array}\right) .
$$

The Hamiltonian associated with problem (4.2) is

$$
H_{\lambda}(X, U, \Lambda):=H(X, U, \Lambda)+(1-\lambda) U^{2}
$$

and consequently the Hamiltonian equations are the same as for (3.1), i.e. (3.7).

For $\lambda=1,(4.2)$ reduces to the problem (3.1) that we want to solve. The interesting point is that for $\lambda \in[0,1[$, problem (4.2) is smooth, $U \mapsto H_{\lambda}(X, U, \Lambda)$ is convex, and the control $U$ is continuous.

\subsubsection{Indirect shooting method to solve the penalized problem}

We consider here that $\lambda \in[0,1[$ is fixed.

Using the analysis performed in Section 3.1, we have that a necessary condition for $(X(),. U()$.$) to be a$ solution of (4.2) is the existence of a non-zero vector $\Lambda():.=(p(),. q()$.$) such that Y:=(X, \Lambda)$ is solution, for $\tau \in] 0, \Upsilon[$, of

$$
\dot{Y}(\tau)=h_{\lambda}(Y(\tau), U(\tau))
$$

where

$$
h_{\lambda}(Y, U)=h(Y, U)+\left(\begin{array}{c}
0 \\
0 \\
(1-\lambda) U^{2} \\
0 \\
0
\end{array}\right)
$$

with the two-point boundary values

$$
X(0)=(0,0,0), X_{1}(\Upsilon)=\Delta, \Lambda_{2}(\Upsilon)=0,
$$

and

$$
U:=\left\{\begin{array}{l}
0 \quad \text { if } U^{*}<0 \\
1 \quad \text { if } U^{*}>1 \\
U^{*} \text { otherwise }
\end{array}\right.
$$


where $U^{*}=-\frac{\nu+\tilde{D} q}{2(1-\lambda)}\left(\right.$ solution of $\left.\frac{\partial H_{\lambda}}{\partial U}\left(U^{*}\right)=0\right)$.

Indirect shooting methods consist in searching a zero of the following shooting function

$$
\begin{aligned}
Z_{\lambda}: \mathbb{R}^{2} & \rightarrow \mathbb{R}^{2} \\
\left(p_{0}, q_{0}\right) & \mapsto\left(Y_{1}(\Upsilon)-\Delta, Y_{5}(\Upsilon)\right),
\end{aligned}
$$

where $Y$ solves $(4.3)$ with $Y(0)=\left(0,0,0, p_{0}, q_{0}\right)$ as initial conditions, and where $U$ is defined in (4.4).

\subsubsection{Multiple indirect shooting method to solve the penalized problem}

For computational efficiency and robustness, we use a multiple shooting method to solve problem (4.2).

Let us introduce the following notation:

- $M$ is the number of subintervals of $[0, \Upsilon]$;

- $\Delta \tau:=\Upsilon / M$ is the length of each of these subintervals;

- $\tau_{i}=i \Delta \tau$, for $i=0,1, \ldots, M$;

- $z_{0}:=\left(0,0,0, p_{0}, q_{0}\right)$ and $z_{i}:=\left(\delta_{i}, \nu_{i}, e_{i}, p_{i}, q_{i}\right)$, for $i=1, \ldots, M-1$;

- for $i=0, \ldots, M-1: z_{\lambda}\left(\tau, \tau_{i}, z_{i}\right)$ is the solution of (4.3) for $\left.\left.\tau \in\right] \tau_{i}, \tau_{i+1}\right]$, with $Y\left(\tau_{i}\right)=z_{i}$ and $U$ defined in (4.4).

Thus, here the multiple indirect shooting method consists in finding a zero of the function $Z_{\lambda, M}$ defined from $\mathbb{R}^{2+5(M-1)}$ to $\mathbb{R}^{5(M-1)+2}$ as follows:

$$
\begin{array}{r}
Z_{\lambda, M}\left(p_{0}, q_{0}, \delta_{1}, \nu_{1}, e_{1}, p_{1}, q_{1}, \ldots, \delta_{M-1}, \nu_{M-1}, e_{M-1}, p_{M-1}, q_{M-1}\right) \\
:=\left(z_{\lambda}\left(\tau_{1}, \tau_{0}, z_{0}\right)-z_{1}, \ldots, z_{\lambda}\left(\tau_{M-1}, \tau_{M-2}, z_{M-2}\right)-z_{M-1},\right. \\
\left.\delta\left(\tau_{M}, \tau_{M-1}, z_{M-1}\right)-\Delta, q\left(\tau_{M}, \tau_{M-1}, z_{M-1}\right)\right) .
\end{array}
$$

The first $5(M-1)$ relations impose the continuity of the states and co-states on the whole trajectory, and the last two relations are the final conditions given by Pontryagin's Minimum Principle.

\subsubsection{Continuation}

The idea of the continuation method is to go smoothly from the solution of (4.2) with $\lambda=0$, which is easy to compute, to the solution of (4.2) with $\lambda \approx 1$.

More precisely, for a fixed number of subintervals $M$, we solve a chain of sub-problems $Z_{\lambda_{k}, M}(\zeta)=0$ for an increasing sequence $\left(\lambda_{k}\right)_{k \in \mathbb{N}}$. This sequence is chosen such that

1. $\lambda_{0}=0$ and it is easy to find a solution $\zeta^{0}$ of $Z_{0, M}(\zeta)=0$;

2. the solution $\zeta^{k}$ of $Z_{\lambda_{k}, M}(\zeta)=0$ is used as a starting point for computing $\zeta^{k+1}$;

3. after $P$ steps, $\zeta^{P}$ should be a good starting point for computing a solution of the relaxed optimal control problem (3.1) (with $\tau_{f}=\Upsilon$ ).

Remark 4.1. In the general case, the above first step (with $\lambda_{0}=0$ ) is not straightforward. For this reason, we propose performing a first continuation on the length of the race track in order to solve initial problem (with $\left.\lambda_{0}=0\right) Z_{0, M}(\zeta)=0$.

We implement the above way of doing in Matlab, using

- the ode113 routine to solve the ordinary differential equations;

- the fsolve routine, with the ('Jacobian' , 'on') option, to find a solution to $Z_{\lambda_{k}, M}(\zeta)=0$. We compute the exact Jacobian of the shooting function using the variational equations.

Figure 4 displays the results. They confirm the existence of singular $\operatorname{arc}(\mathrm{s})$. Indeed, in the case of the flat race track, there is one singular arc for $\tau$ between around 0.15 and 0.65 . In the general case, it remains difficult to evaluate the number of singular arcs. 

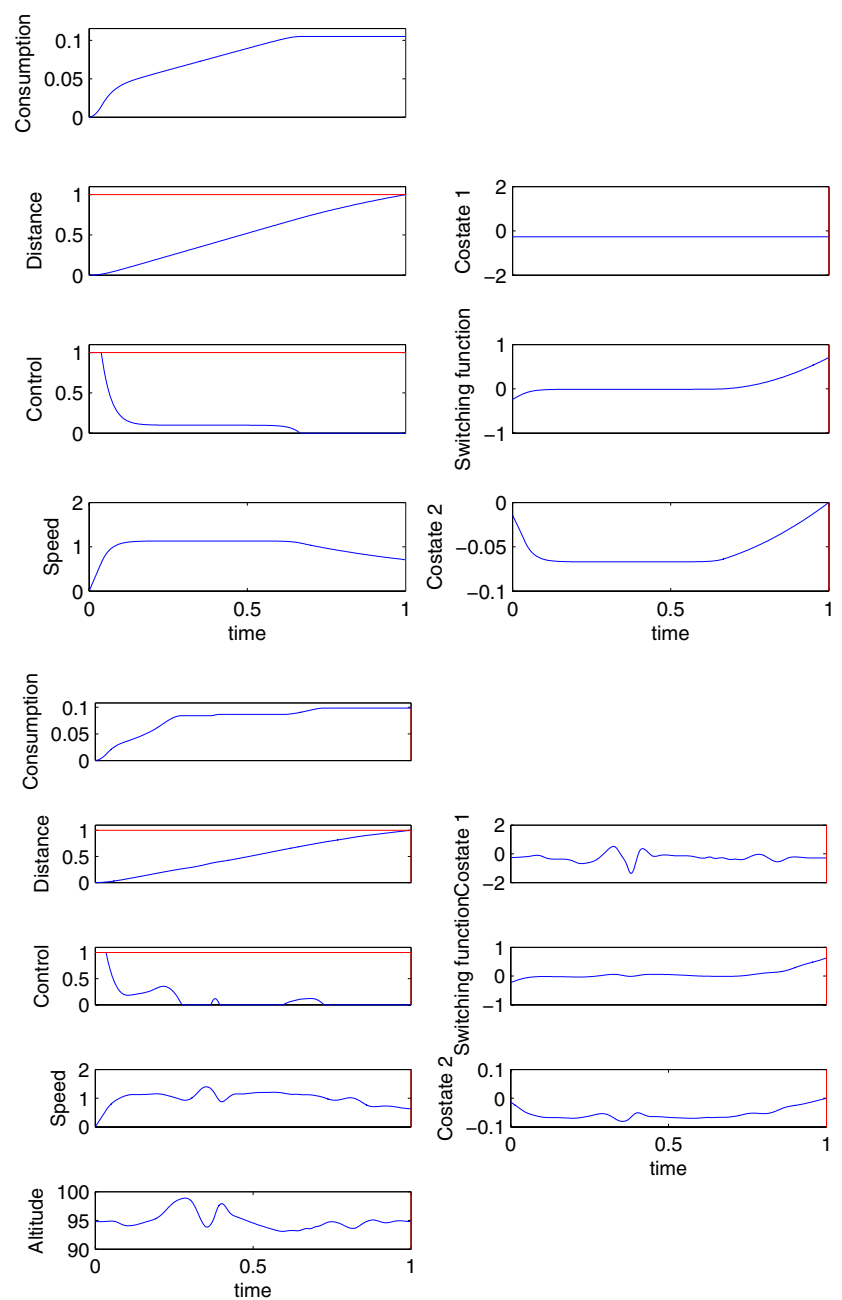

FigURE 4 . Results obtained by the quadratic penalty continuation method with $M:=20$ subintervals of $[0, \Upsilon]$. Top: flat race track. Bottom: general case.

\subsection{Accurate solution of the relaxed optimal control problem with $\tau_{f}=\Upsilon$ in the case of a flat race track}

Thanks to the preceding numerical experiments, the structure of the optimal control for problem (3.1) on a flat race track seems to be of type "on-singular-off". Let us now determine precisely the switching times. For this purpose, we use once more a multiple indirect shooting method.

Let us introduce the following notation:

- $z_{\text {on }}(\tau)$ denotes the solution of (3.7), for $\left.\left.\tau \in\right] 0, \tau_{1}\right]$ with $Y(0)=z_{0}$ and $U=1$;

- $z_{\text {sing }}(\tau)$ is the solution of (3.7) for $\left.\left.\tau \in\right] \tau_{1}, \tau_{2}\right]$ with $Y\left(\tau_{1}\right)=z_{1}$ and $U$ is as defined in (3.6);

- $z_{\text {off }}(\tau)$ is the solution of (3.7) for $\left.\left.\tau \in\right] \tau_{2}, \Upsilon\right]$ with $Y\left(\tau_{2}\right)=z_{2}$ and $U=0$.

The multiple indirect shooting method therefore consists in finding a zero of the function $Z$ defined from $\mathbb{R}^{2+1+5+1+5}$ to $\mathbb{R}^{5+5+2+2}$ as follows:

$$
\begin{array}{r}
Z\left(p_{0}, q_{0}, \tau_{1}, \delta_{1}, \nu_{1}, e_{1}, p_{1}, q_{1}, \tau_{2}, \delta_{2}, \nu_{2}, e_{2}, p_{2}, q_{2}\right):=\left(z_{\text {on }}\left(\tau_{1}\right)-z_{1}, z_{\text {sing }}\left(\tau_{2}\right)-z_{2}\right. \\
\left.\nu_{1}+\tilde{D} q_{1}, 3 \tilde{B} \nu_{1}^{2}+\tilde{C} k_{0}-\tilde{A} \tilde{D} p_{1}, \delta_{\text {off }}(\Upsilon)-1, q_{\text {off }}(\Upsilon)\right) .
\end{array}
$$



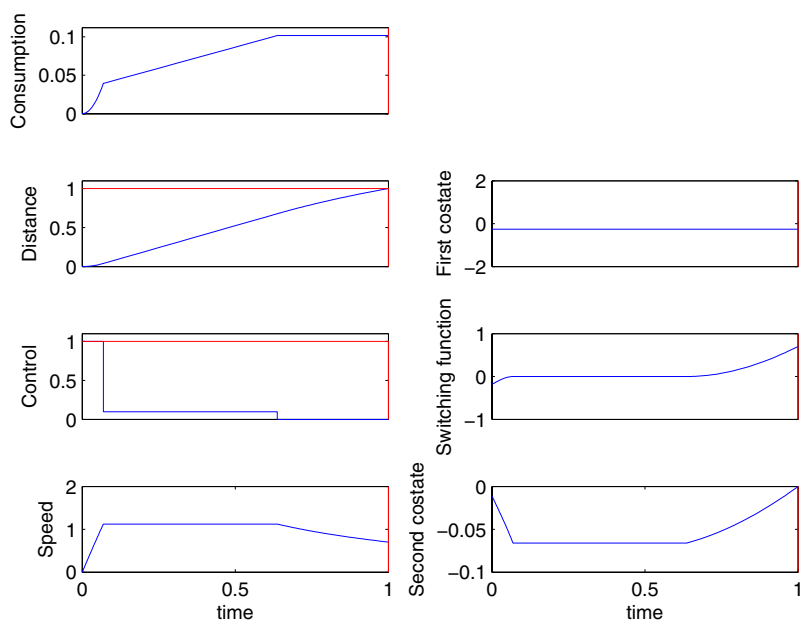

FiguRE 5. Results obtained by a multiple indirect shooting method on problem (3.1) in the simplified case.

The first 10 relations impose the continuity of the states and co-states on the whole trajectory. The last two relations are the final conditions given by Pontryagin's Minimum Principle. The other two relations correspond to the zero values at the first switching time of the switching function and its first derivative. As for starting points, we have used approximate values obtained at the end of the continuation process.

Using Matlab and the same routines as before (but with the ('Jacobian' , 'off') option), we obtain the results displayed in Figure 5. These give us precise values for the switching times and optimal consumption:

$$
\tau_{1}=0.069, \quad \tau_{2}=0.6368 \text { and } \quad e_{\mathrm{off}}(\Upsilon)=0.10165
$$

Remark 4.2. In the simplified case of a flat race track, since the function $\tilde{\mathcal{C}}$ only depends on $\nu$, the singular control is given by (3.6):

$$
U(\tau)=-\frac{1}{\tilde{D}}\left(\tilde{B} \nu(\tau)^{2}+\tilde{\mathcal{C}}(\delta, \nu)\right)
$$

This implies that the second component of the function $h$ used in (3.7) is zero. Consequently, the speed $\nu$ and the control are constant between $\tau_{1}$ and $\tau_{2}$. This can be seen in Figure 5 .

\subsection{A first heuristics to solve the original intrinsically bang-bang optimal control problem}

In the preceding sections, we have studied a relaxed optimal control problem in which the control is allowed to take any value between 0 and 1 . However, our original problem is concerned with purely $0-1$ solutions. In this section, we describe a first heuristics that allows us to find purely bang-bang solutions to the original problem (2.1).

First, we assume that there is a given number $N$ of switching times. Let us introduce the following notation:

- $0=\tau_{0} \leq \tau_{1} \leq \tau_{2} \leq \ldots \leq \tau_{N} \leq \tau_{N+1}=\tau_{f} \leq \Upsilon$;

- $I_{i}:=\left[\tau_{i-1}, \tau_{i}\right]$, for $i=1, \ldots, N+1$;

- for $i=1, \ldots, N+1$,

$$
U_{i}= \begin{cases}1 & \text { for odd } i \\ 0 & \text { for even } i\end{cases}
$$



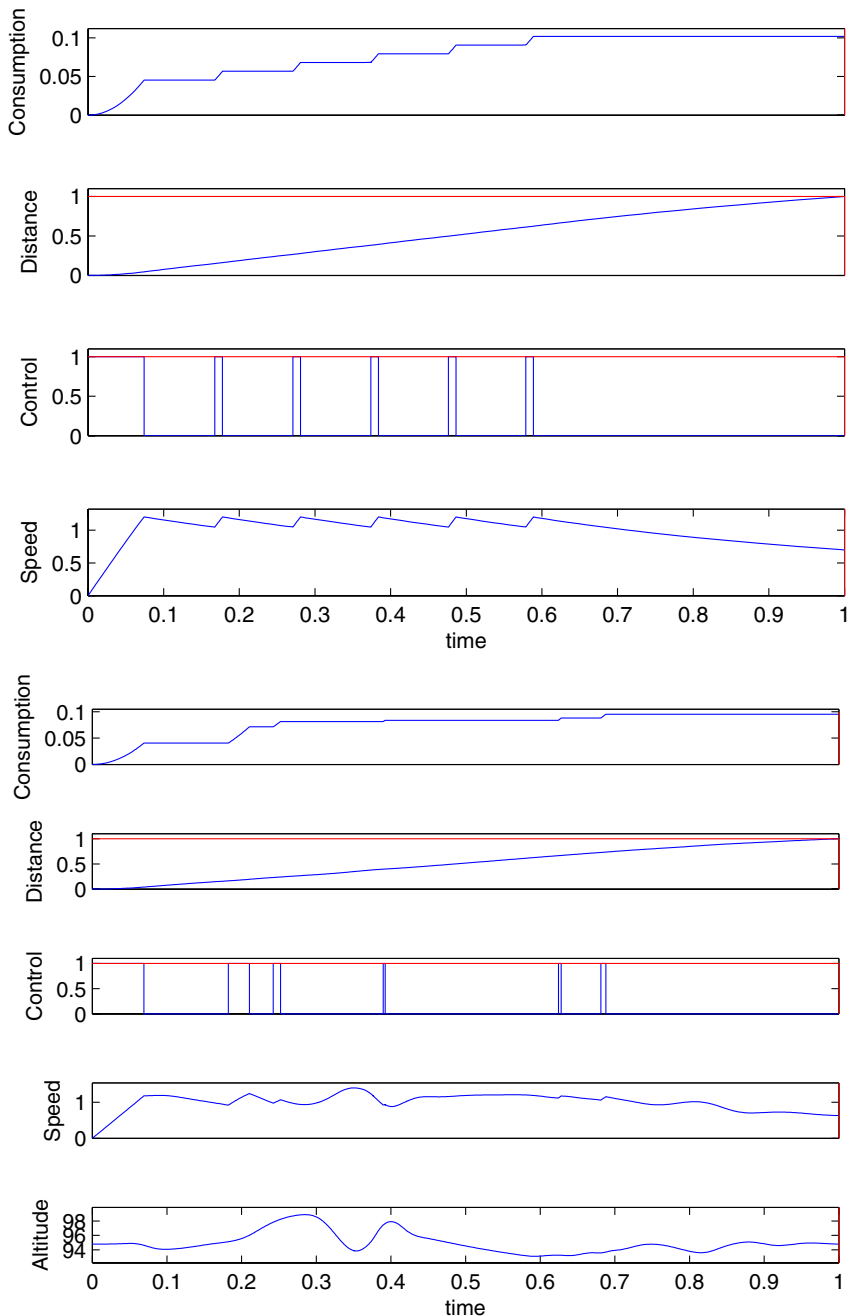

FiguRE 6. Results obtained by the nonlinear programming approach (4.5) with $N=11$ switching times into $\left[0, \tau_{f}\right]$. Top: flat race track. Bottom: general case.

- $X(\tau, 1)$ is the solution of $(2.2)$ on $I_{1}$ with $U(\tau) \equiv U_{1}$ and $X\left(\tau_{0}, 1\right)=(0,0,0)$;

- $X(\tau, i)$ is the solution of $(2.2)$ on $I_{i}$ with $U(\tau) \equiv U_{i}$ and $X\left(\tau_{i-1}, i\right)=X\left(\tau_{i-1}, i-1\right)$ for $i=2, \ldots, N+1$.

We consider the following problem:

$$
\begin{aligned}
& \text { Minimize } X_{3}\left(\tau_{N+1}, N+1\right) \\
& \text { subject to } 0=\tau_{0} \leq \tau_{1} \leq \tau_{2} \leq \ldots \leq \tau_{N} \leq \tau_{N+1}=\tau_{f} \leq \Upsilon, \\
& X_{1}\left(\tau_{N+1}, N+1\right)=\Delta
\end{aligned}
$$

where the unknowns are the $N+1$ values of $\tau_{i}$.

We tackle problem (4.5) with the interior-point fmincon (with the integrator ode113) routine of Matlab for $N=1,2, \ldots, 19$. We perform fmincon for different random initial guesses of the unknowns, and this for each of considered values of $N$. An example of results we can obtain with this method is displayed in Figure 6 .

In both cases, we observe in Figure 7 that the cost of the solution of (4.5) decreases when the number of switching times $N$ increases. When the race track is flat, this cost seems to converge to the optimal cost of the 

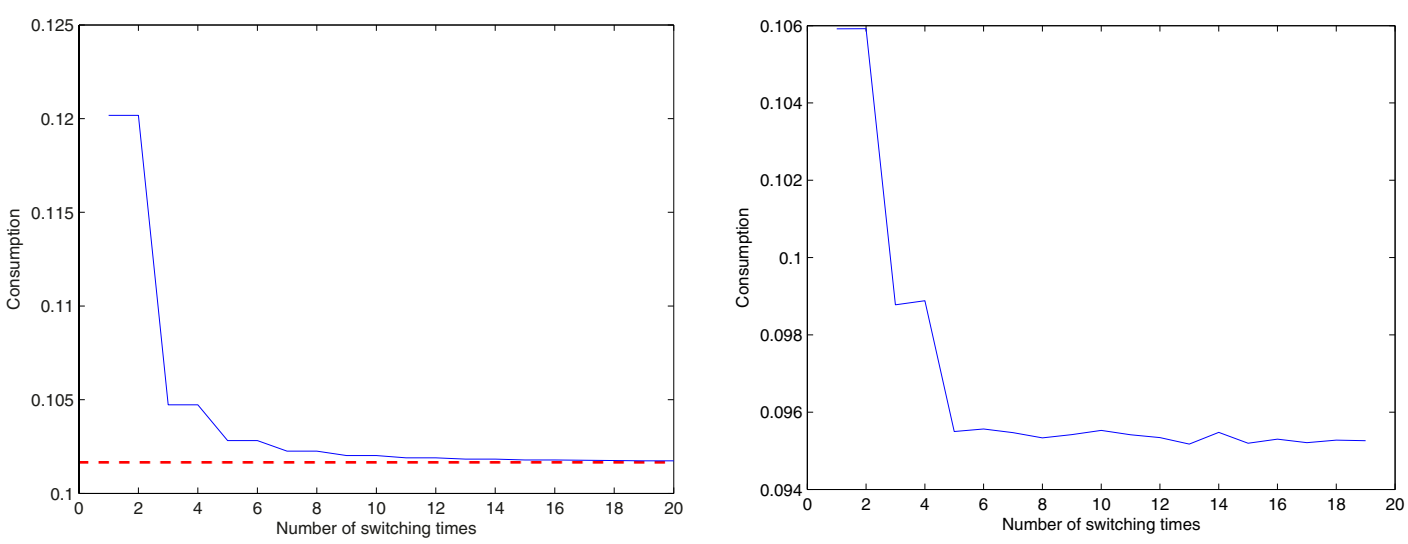

Figure 7. Decreasing of the consumption (plain) with the number of switching times and comparison with the optimal consumption of the relaxed problem (dashed). Top: flat race track. Bottom: general case.
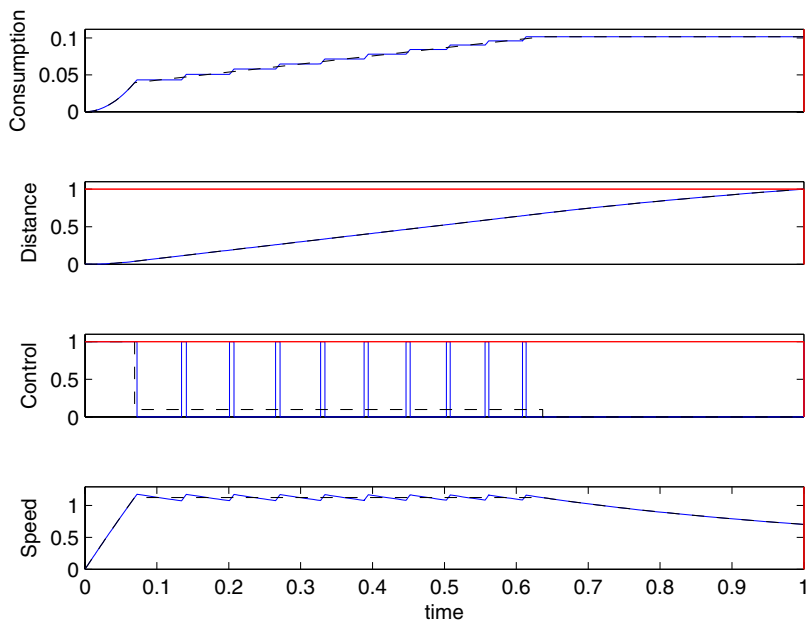

FiguRE 8. Comparison, for the case of a flat race track, between the solution of Problem (4.5) (plain) and the accurate solution of the relaxed problem (dashed).

relaxed problem. In the general case, the existence of local minima may explain the small fluctuations we can observe on the cost.

A comparison is made in Figure 8 between the results obtained with this heuristics for $N=20$ and the singular arc obtained for the relaxed problem when the race track is flat. We can observe that the consumption and the speed obtained with these two different methods are very similar. This is obtained thanks to an accumulation of switching times during the singular arc.

Finally, we show in Figure 9 the impact of the final time on the criterion. As expected, the cost of the solution of (4.5) increases when the final time decreases.

With this methodology, we confirm once more the intuitive fact that $\tau_{f}=\Upsilon$. However, these results are not completely satisfactory because we have no guarantee of optimality. It would be interesting to study whether the guaranteed global optimization techniques used in [7] are applicable here. 


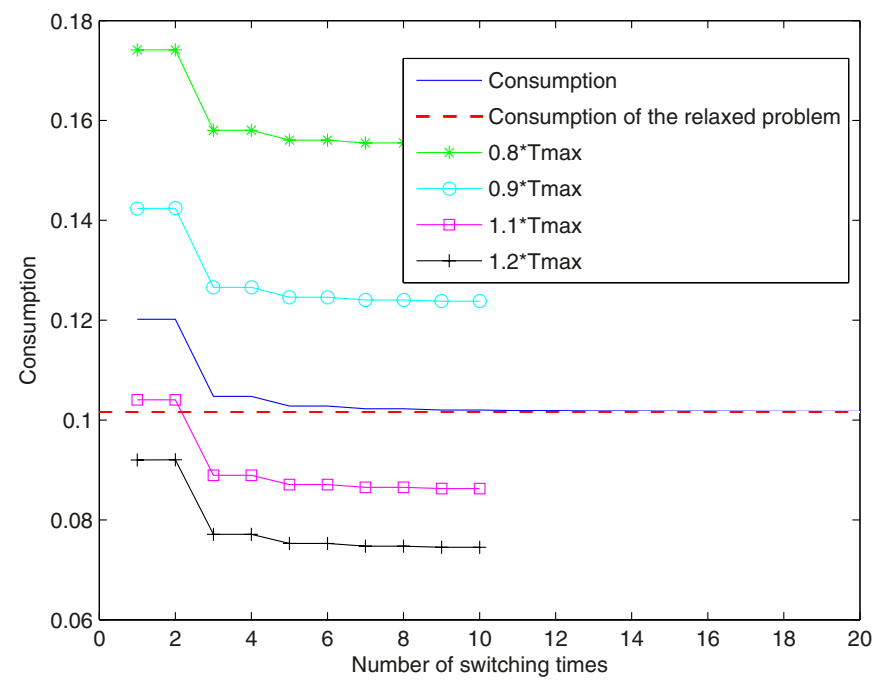

FiguRE 9. Impact of the final time on the consumption, in the case of the flat race track.

\section{Conclusions AND PERSPECTIVES}

We have studied the behaviour of an intrinsically bang-bang optimal control problem by considering its continuous relaxation. Using different methodologies (nonlinear programming and indirect shooting methods), we have numerically established that the relaxed problem involves singular arcs. Finally, we could recover purely bang-bang solutions of the original problem of minimizing the fuel consumption of a vehicle from the Shell Eco-marathon using a nonlinear programming approach.

It would be interesting to extend our study in several ways. First, it could be interesting to study other techniques such as branch-and-bound based global optimization methods using interval analysis [7]. Then, in this paper, the race track is considered as a straight line. We should study the influence of the curves of the race track on the numerical results. Another issue involves considering an engine torque that depends on the vehicle speed $v$.

Acknowledgements. This study was prompted by Yves Mégret, the leader of the Toulouse Ingénierie Mécanique team. The author is also thankful to Bonnans [2] and Epenoy [1] for interesting discussions about logarithmic continuation, Jean-Baptiste Hiriart-Urruty for his encouragement, Marcel Mongeau and Aude Rondepierre for their help, Sager [9] for a test of his code, and Emmanuel Trélat for more discussions about the continuation approach (quadratic versus logarithmic).

\section{REFERENCES}

[1] R. Bertrand and R. Epenoy, New smoothing techniques for solving bang-bang optimal control problems - Numerical results and statistical interpretation. Optim. Control Appl. Methods 23 (2002) 171-197.

[2] J.F. Bonnans and F. Silva, Error estimates for the logarithmic barrier method in linear quadratic stochastic optimal control problems. Technical Report, INRIA RR 7455 (2010).

[3] F. Bonnans, P. Martinon and E. Trélat, Singular arcs in the generalized Goddard's problem. J. Optim. Theory Appl. 139 (2008) 439-461.

[4] J.-B. Hiriart-Urruty, Les mathématiques du mieux faire, Ellipses. La commande optimale pour les débutants 2 (2008).

[5] L.M. Hocking, Optimal control, An introduction to the theory with applications, Oxford Applied Mathematics and Computing Science Series. The Clarendon Press Oxford University Press (1991).

[6] A. Locatelli, Optimal control, An introduction. Birkhäuser Verlag (2001). 
[7] A. Merakeb, F. Messine and M. Aidene, On minimizing the energy consumption of an electrical vehicle. Research Report RT-APO-11-4, IRIT, Université Paul Sabatier, Toulouse (2011).

[8] H.M. Robbins, A generalized Legendre-Clebsch condition for the singular cases of optimal control. IBM J. Research Devel. 11 (1967) 361-372.

[9] S. Sager, H. G. Bock, M. Diehl, G. Reinelt and J. P. Schlöder, Numerical methods for optimal control with binary control functions applied to a Lotka-Volterra type fishing problem, in Recent advances in optimization, Lect. Notes Econ. Math. Syst. 563 (2006) 269-289.

[10] J. Stoer and R. Bulirsch, Introduction to numerical analysis. Translated from the German by R. Bartels, W. Gautschi and C. Witzgall, Springer-Verlag (1980).

[11] E. Trélat, Contrôle optimal, Mathématiques Concrètes. Vuibert, Paris. Théorie E applications (2005). 